Japan. J. Math.

Vol. 16, No. 1, 1990

\title{
Remarks on the $j$-codegree of vector bundles
}

\author{
By Hideaki Ōshima \\ Dedicated to Professor Hirosi Toda on his 60th birthday
}

(Received September 26, 1988)

\section{§1. Introduction}

Let $V$ be an orientable vector bundle over a connected finite CW-complex $X$ with the Thom space $T(V)$. The codegree cd $(V)$ of $V$ [14] is the order of the cokernel of $i^{*}: \pi_{s}^{n}(T(V)) \rightarrow \pi_{s}^{n}\left(S^{n}\right)$, where $n=\operatorname{dim} V, \pi_{s}^{*}$ is the stable cohomotopy and $i: S^{n}=T\left(\left.V\right|_{x}\right) \subset T(V)$ for any point $x$ of $X$. The computation of $c d(V)$ has important applications (e.g., James numbers) but it is difficult in general. Other more computable codegrees can be obtained using $K O$-theory and versions of image $J$-theory $j, j^{\prime}$ instead of $\pi_{s}^{*}$ :

$$
\operatorname{cd}^{K o}(V)\left|\operatorname{cd}^{j^{\prime}}(V)\right| \mathrm{cd}^{j}(V) \mid \operatorname{cd}(V) .
$$

Here $a \mid b$ means that $b$ is a multiple of $a$, and each codegree is much easier to compute than a codegree on the right hand side. Precise definitions will be given in $\S 2$.

The purpose of this note is to study relations of $\operatorname{cd}^{j^{\prime}}(V)$ and $\operatorname{cd}^{j}(V)$. We give a sufficient condition and a counter example to the equation $\operatorname{cd}^{j^{\prime}}(V)=$ $\mathrm{cd}^{j}(V)$. Our results are the following two theorems.

THEOREM 1.1. Let $V$ be a vector bundle over a connected finite $C W$-complex $X$. Then $\mathrm{cd}^{j}(V) \mid 2 \mathrm{~cd}^{j^{\prime}}(V)$. If $H^{1}\left(X ; Z_{2}\right)=0$ or the second Stiefel Whitney class $w_{2}(V)$ of $V$ vanishes, then $\operatorname{cd}^{j^{\prime}}(V)=\operatorname{cd}^{j}(V)$.

TheOREm 1.2. Let $L^{4}(m)$ be the 4-skeleton of the standard lens space $B Z_{m}$ $=S^{\infty} / Z_{m}$. Let $H_{4}^{\prime}$ be the induced bundle of the canonical complex line bundle over the complex projective space $B S^{1}=S^{\infty} / S^{1}$ by the composition $L^{4}(m) \subset B Z_{m}$ $\rightarrow B S^{1}$. Then we have

(1) $\operatorname{cd}^{j}(V)=\mathrm{cd}\left(V^{j}\right)$ for every vector bundle $V$ over $L^{4}(m)$.

(2) If $n \equiv 3(\bmod 4)$ and $m \equiv 8(\bmod 16)$, then $\operatorname{cd}_{2}^{K o}\left(n H_{4}^{\prime}\right)=1, \operatorname{cd}_{2}^{j^{\prime}}\left(n H_{4}^{\prime}\right)=2$ and $\operatorname{cd}_{2}^{j}\left(n H_{4}^{\prime}\right)=3$.

Here $\operatorname{cd}_{p}^{K O}(V), \mathrm{cd}_{p}^{j^{\prime}}(V)$ and $\mathrm{cd}_{p}^{j}(V)$ are the exponents of the prime $p$ in the prime decompositions of respective numbers. 
Example 3.5 below shows that the assumption of Theorem 1.1 is only a sufficient condition to $\mathrm{cd}^{j}(V)=\mathrm{cd}^{j^{\prime}}(V)$.

No counter example to the equation $\operatorname{cd}^{j}(V)=\mathrm{cd}(V)$ is known, while it holds for a suitable family of bundles [5]. Codegrees can be defined also for spherical fibrations and it is known by Crabb-Knapp, Nishida and others that for each prime $p$ there exists a spherical fibration $V$ such that $\operatorname{cd}_{p}(V)$ $=1$ and $\operatorname{cd}_{p}^{j}(V)=0$.

In this note all generalized cohomology theories are reduced.

This note is organized as follows. In $\S 2$, we recall the definition of codegrees. In $\S 3$, we collect elementary properties of codegrees. In $\S 4$, we prove Theorem 1.1. In $\S 5$, we prove Theorem 1.2 as a corollary to Theorems 5.4 and 5.5.

The part of Theorem 1.1 containing the assumption $w_{2}(V)=0$ is due to K. Knapp to whom I am greatly indebted for allowing me to include it in this note. I would like also to thank G. Nishida for giving me information on the codegrees of spherical fibrations.

\section{§ 2. Definition of codegrees}

Let $E=\left\{E_{n}\right\}$ be an $\Omega$-spectrum such that $E_{n}$ is a CW-complex for every $n \in Z$. We denote the $(k-1)$-connective covering spectrum for $E$ by $E[k, \infty)$, that is, $E[k, \infty)$ is a $(k-1)$-connected $\Omega$-spectrum with a map of $\Omega$-spectra $f$ : $E[k, \infty) \rightarrow E$ such that $f_{*}: \pi_{i}(E[k, \infty)) \rightarrow \pi_{i}(E)$ is an isomorphism for $i \geqq k$. More precisely speaking, $E[k, \infty)=\left\{F_{n}\right\}$ and $f$ are constructed as follows: if $n \geqq-k+1$, then $F_{n}=E_{n}[n+k, \infty)$, the $(n+k-1)$-connective fibering over the path component $E_{n}^{\prime}$ containing the base point of $E_{n}$, and $f_{n}: F_{n} \rightarrow E_{n}^{\prime} \subset E_{n}$ is the composite of the projection and the inclusion; if $n<-k+1$, then $F_{n}=$ $\Omega^{-n-k+1} F_{-k+1}$ and $f_{n}: F_{n}=\Omega^{-n-k+1} F_{-k+1}=\Omega^{-n-k+1} E_{-k+1} \simeq E_{n}$, where the last homotopy equivalence is the structure map of $E$.

Let $p$ be a prime number. We write $\nu_{p}(b)$ for the exponent to which the prime $p$ occurs in the decomposition of a rational number $b$ into prime powers. We set $\nu_{p}(0)=+\infty$. For each prime $p$ we choose and fix an integer $u$ such that $u \equiv 3(\bmod 8)$ if $p=2$ and $u$ is an odd integer which generates the group of units of $Z / p^{2} Z$ if $p \neq 2$. Let $\psi_{*}^{u}: K F_{(p)} \rightarrow K F_{(p)}$ be the stable Adams operation on the $p$-localized $K F$-theory, and let $\psi_{n}^{u}: K F_{n(p)} \rightarrow K F_{n(p)}$ be its $n$-th term. Here $F$ denotes the field $\boldsymbol{R}$ of real numbers or the field $\boldsymbol{C}$ of complex numbers. As usual we write $K O$ and $K$ instead of $K \boldsymbol{R}$ and $K \boldsymbol{C}$, respectively. Let $d$ be the dimension of $F$ over $\boldsymbol{R}$. We can lift $\psi_{*}^{u}-1$ uniquely to $\psi_{*}^{u}-1: K F[0, \infty)_{(p)} \rightarrow K F[0, \infty)_{(p)}$ and moreover to $\psi: K F[0, \infty)_{(p)}$ $\rightarrow K F[4 / d, \infty)_{(p)}$ (see May [11; p. 121]). We denote the fibres of $\psi_{*}^{u}-1$ and $\psi$ by $j F^{\prime}\langle p\rangle$ and $j F\langle p\rangle$, respectively. They are $\Omega$-spectra. We write $j^{\prime}\langle p\rangle$ and 
$j\langle p\rangle$ instead of $j \boldsymbol{R}^{\prime}\langle p\rangle$ and $j \boldsymbol{R}\langle p\rangle$. If confusion does not occur then we omit the notation $\langle p\rangle$. The 0 -th term of the fibration

$$
j\langle p\rangle \longrightarrow K O[0, \infty)_{(p)} \stackrel{\psi}{\longrightarrow} K O[4, \infty)_{(p)}
$$

is the fibration

$$
Z_{(p)} \times J_{(p)} \stackrel{1 \times i}{\longrightarrow} Z_{(p)} \times B O_{(p)} \longrightarrow \operatorname{BSpin}_{(p)}
$$

which is obtained naturally from the fibration

$$
J \stackrel{i}{\longrightarrow} \mathrm{BO} \stackrel{\Psi}{\longrightarrow} \mathrm{BSpin},
$$

where $\psi$ is the unique lifting of $\psi^{u}-1: B O \rightarrow B O$.

By the solution of Adams conjecture, we have a commutative diagram

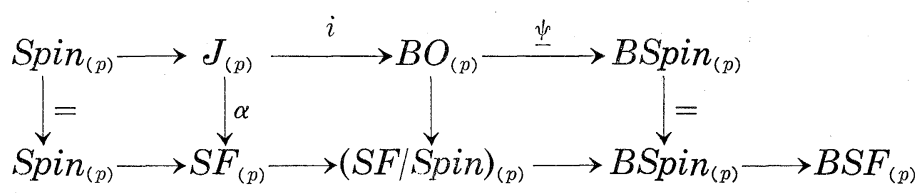

where $B S F$ is the classifying space of orientable spherical fibrations. The unit of $K O_{(p)}$ lifts uniquely to $j\langle p\rangle$ so that we have an infinite loop map

$$
e: Q\left(S^{0}\right) \longrightarrow Z_{(p)} \times J_{(p)}
$$

which induces the Hurewicz homomorphism for the 0 -dimension of $j\langle p\rangle$ cohomology, where $Q\left(S^{0}\right)=\lim _{k} \Omega^{k} S^{k}$. Let $Q_{m}\left(S^{0}\right)$ be the connected component containing maps of degree $m$. Then $S F=Q_{1}\left(S^{0}\right)$, and $e$ carries $Q_{m}\left(S^{0}\right)$ into $\{m\} \times J_{(p)}$. We use the notation $e_{m}$ for the restriction of $e$ to $Q_{m}\left(S^{0}\right)$ and its p-localization. The following is well-known (see e.g. May [11; Chapter V]).

TheOREm 2.1. The composite $e_{1} \alpha: J_{(p)} \rightarrow Q_{1}\left(S^{0}\right)_{(p)} \rightarrow\{1\} \times J_{(p)}$ is a weak homotopy equivalence.

We deform $e_{m}$ to $e_{m}^{\prime}$ by the commutative square

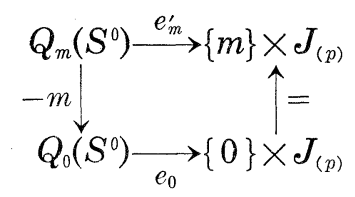

where $-m$ denotes the sum with a map of degree $-m$ and the right vertical map is the natural identification. The function $e^{\prime}=\left\{e_{m}^{\prime}\right\}: Q\left(S^{0}\right) \rightarrow Z_{(p)} \times J_{(p)}$ is continuous and satisfies the relation $(1 \times i) e^{\prime} \simeq(1 \times i) e$. Moreover 2.1 with $e_{1}$ replaced by $e_{1}^{\prime}$ is true. Thus we have 
Corollary 2.2. Let $X$ be a connected finite $C W$ complex. Then the composition

$$
j\langle p\rangle^{0}(X) \stackrel{\alpha_{*}}{\longrightarrow}\left[X, Q_{1}\left(S^{0}\right)_{(p)}\right] \stackrel{(-1)_{*}}{\cong}\left[X, Q_{0}\left(S^{0}\right)_{(p)}\right] \stackrel{e_{0 *}}{\longrightarrow} j\langle p\rangle^{0}(X)
$$

is a bijection, where $[X, Y]$ is the set of homotopy classes of continuous maps from $X$ to $Y$. In particular the Hurewicz homomorphism $e_{0 *}: \pi_{s}^{0}(X)_{(p)} \rightarrow$ $j\langle p\rangle^{\circ}(X)$ is a surjection.

Note that the middle bijection in 2.2 is not necessarily a homomorphism, but is so if $X$ is a suspension.

By using the information above we can show

Proposition 2.3. The coefficient group of $j\langle p\rangle$ is carried isomorphically by $(-1)_{*} \alpha_{*}$ to the image of the classical J-homomorphism at $p \neq 2$ and to that with $\mu, \eta \mu$-family of Adams at $p=2$, where $\eta$ is the generator of the stable 1 stem $\pi_{1}^{s}\left(S^{0}\right)=Z_{2}$. In particular the Hurewicz homomorphism $\pi_{s}^{0}\left(S^{n}\right)_{(p)} \rightarrow$ $j\langle p\rangle^{0}\left(S^{n}\right)$ is an isomorphism for $n \leqq 5$.

Let $E$ be a generalized reduced cohomology theory (i.e. a spectrum) such that

$$
E^{0}\left(S^{0}\right) / \operatorname{Tor} \subset \boldsymbol{Q}
$$

where Tor denotes the torsion subgroup and $\boldsymbol{Q}$ is the group of rational numbers.

EXAMPLE 2.4. The following spectra satisfy the above condition: $\mathscr{S}$ (the sphere spectrum), $\mathscr{K}(Z, 0)$ (the Eilenberg MacLane spectrum with integra] coefficients), $K F, K F[0, \infty), j F, j F^{\prime}, M U$ (the complex cobordism spectrum), $B P$ (the Brown Peterson spectrum). Indeed all of these spectra except $j R^{\prime}\langle 2\rangle$ satisfy $E^{0}\left(S^{0}\right)=Z$ or $Z_{(p)}$, while $j R^{\prime}\langle 2\rangle^{0}\left(S^{0}\right)=Z_{(2)} \oplus Z_{2}$.

Of course $\pi_{s}^{n}(-)=\mathscr{S}^{n}(-)$.

Let $V$ be an $n$-dimensional vector bundle over a connected finite CWcomplex $X, x \in X$, and let $i: S^{n}=T\left(\left.V\right|_{x}\right) \rightarrow T(V)$ be the inclusion map. Consider the homomorphisms

$$
E^{n}(T(V)) \stackrel{i^{*}}{\longrightarrow} E^{n}\left(S^{n}\right) \stackrel{q}{\longrightarrow} E^{n}\left(S^{n}\right) / \text { Tor }
$$

where $q$ is the quotient homomorphism. Then the $E$-codegree of $V, \operatorname{cd}(V ; E)$, is defined to be 0 if $q i^{*}=0$, and to be the cardinal number of the cokernel of $q i^{*}$ if $q i^{*} \neq 0$. Set $\operatorname{cd}(V)=\operatorname{cd}(V ; \mathscr{S})$.

LEMma 2.5. (1) $\mathrm{cd}(V ; E)$ does not depend on the choice of $x$. 
(2) $\operatorname{cd}(V \oplus m ; E)=\mathrm{cd}(V ; E)$ and $\operatorname{cd}(m ; E)=1$, where $m$ is a trivial $m$ dimensional vector bundle.

(3) $\operatorname{cd}(V ; E)=\operatorname{cd}(V ; E[0, \infty))$, where $E[0, \infty)$ is a $(-1)$-connective covering of an $\Omega$-spectrum representing $E$.

(4) If $E$ is a spectrum of Example 2.4, then $\mathrm{cd}(V ; E) \mid \mathrm{cd}(V)$.

(5) Suppose that $E^{0}\left(S^{0}\right) /$ Tor $\neq 0$ and $E^{k}\left(S^{0}\right)$ is a torsion for every $k<0$. Then the following are equivalent.

$(5-1) \quad \mathrm{cd}(V ; E)=0$.

(5-2) $E^{n}(T(V))$ is a torsion.

(5-3) $V$ is non-orientable.

Proof. (1) and (2) are trivial. (3) follows from 4.6 below.

If $E$ is a spectrum of Example 2.4, then there exists a map of spectra $\iota$ : $\mathscr{S} \rightarrow E$ such that the induced homomorphism $\pi_{0}(\mathscr{S}) \rightarrow \pi_{0}(E) /$ Tor or its localization $\pi_{0}(\mathscr{S})_{(p)} \rightarrow \pi_{0}(E) /$ Tor is an isomorphism. Thus (4) follows.

To prove (5), we can assume, by (3), that $E$ is (-1)-connected and, by (1), that the base point $x$ is a vertex of $X$. Then (5) can be proved using the exact sequence of the pair $\left(T(V), S^{n}\right)$ and the Atiyah-Hirzebruch spectral sequence to $E^{n}(T(V))$. We omit the details.

REMARK 2.6. (1) The spectra $\mathscr{S}, j F^{\prime}$ and $j F$ satisfy the assumption of $2.5(5)$.

(2) If $E$ is a multiplicative theory and $V$ is $E$-orientable, then cd $(V ; E)$ $=1$.

We use the following notations:

$$
\begin{aligned}
\operatorname{cd}_{p}(V) & =\nu_{p}(\operatorname{cd}(V)) ; \\
\operatorname{cd}_{p}^{j F}(V) & =\nu_{p}(\operatorname{cd}(V ; j F\langle p\rangle)) ; \\
\operatorname{cd}_{p}^{j F^{\prime}}(V) & =\nu_{p}\left(\operatorname{cd}\left(V ; j F^{\prime}\langle p\rangle\right)\right) ; \\
\operatorname{cd}^{j F}(V) & =\Pi_{p} \operatorname{cd}(V ; j F\langle p\rangle) ; \\
\operatorname{cd}^{j F^{\prime}}(V) & =\Pi_{p} \operatorname{cd}\left(V ; j F^{\prime}\langle p\rangle\right) .
\end{aligned}
$$

We use also the notations $\operatorname{cd}_{p}^{j}(V), \operatorname{cd}^{j}(V), \operatorname{cd}_{p}^{j^{\prime}}(V)$ and $\operatorname{cd}^{j^{\prime}}(V)$ when $F=\boldsymbol{R}$. Notice that $\operatorname{cd}(V ; j F\langle p\rangle), \operatorname{cd}\left(V ; j F^{\prime}\langle p\rangle\right)$ are powers of $p$ or zero and that $\mathrm{cd}^{j F}(V), \mathrm{cd}^{j F^{\prime}}(V)$ are finite integers by 2.5(4), and their exponents of $p$ are $\operatorname{cd}_{p}^{j F^{\prime}}(V), \operatorname{cd}_{p}^{j F^{\prime}}(V)$, respectively. By definition we have

LEMMA 2.7. If $V$ is orientable and of dimension $n$, then

$$
\begin{gathered}
\operatorname{cd}_{p}^{j F}(V)=\operatorname{Min}\left\{e \geqq 0 ; i^{*} y=p^{e} \in Z_{(p)}=K F[0, \infty)^{n}(T(V \mid x))_{(p)}\right. \\
\text { for some } y \in \operatorname{Ker}(\psi)\}, \\
\operatorname{cd}_{p}^{j F^{\prime}}(V)=\operatorname{Min}\left\{e \geqq 0 ; i^{*} y=p^{e} \in Z_{(p)}=K F[0, \infty)^{n}(T(V \mid x))_{(p)}\right. \\
\text { for some } \left.y \in \operatorname{Ker}\left(\psi_{n}^{u}-1\right)\right\},
\end{gathered}
$$


where Min and Ker mean the minimum and the kernel, respectively.

By 2.3, we have

Lemma 2.8. If $X=S^{n} \cup e^{n+1} \cup \cdots \cup e^{n+i}$, then the Hurewicz homomorphism $\pi_{s}^{n}(X) \rightarrow j\langle p\rangle^{n}(X)$ is an isomorphism for $i<6$ and a surjection for $i=6$, where $e^{k}$ is a cell of dimension $k$.

Proposition 2.9. If $V$ is an $n$-dimensional vector bundle over $X=e^{0} \cup e^{1}$ $\cup \cdots \cup e^{i}$ with $i \leqq 6$, then $\mathrm{cd}^{j}(V)=\mathrm{cd}(V)$.

Proof. Since $T(V)=S^{n} \cup e^{n+1} \cup \cdots \cup e^{n+i}$, the result follows from 2.8 .

Now we recall from Crabb-Knapp [6] other lower bound for $\mathrm{cd}(V)$. If $V$ is non-orientable, then we set $\operatorname{cd}^{K F}(V)=0$. In case $V$ is orientable, we denote by $U_{H}(V) \in H^{n}(T(V) ; Z)$ a Thom class of $V$ and define

$$
\begin{aligned}
& \operatorname{cd}^{K F}(V)=\operatorname{Min}\left\{m>0 ; m U_{H}(V) \in \operatorname{Im}\left(\operatorname{ch}_{F}: K F^{n}(T(V)) \longrightarrow H^{*}(T(V) ; \boldsymbol{Q})\right)\right\}, \\
& \operatorname{cd}_{p}^{K F}(V)=\nu_{p}\left(\operatorname{cd}^{K F}(V)\right),
\end{aligned}
$$

where Im means the image and $\mathrm{ch}_{F}$ is the Chern character or the Pontrjagin character according as $F$ is $\boldsymbol{C}$ or $\boldsymbol{R}$. The following commutative diagram implies that $\operatorname{cd}^{K F^{\prime}}(V)$ is well-defined and satisfies $\mathrm{cd}(V ; K F)\left|\mathrm{cd}^{K F}(V)\right| \mathrm{cd}(V)$.

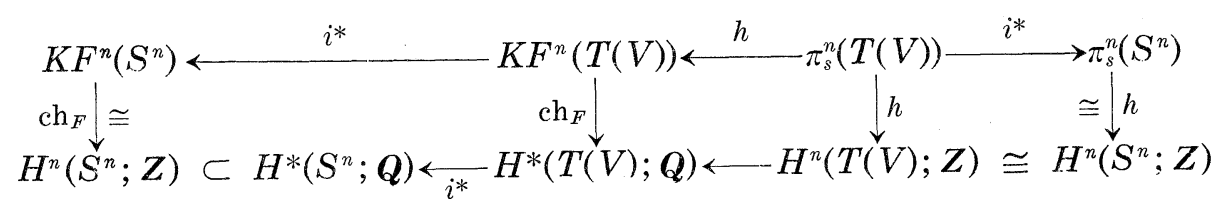

Here $h$ are the Hurewicz homomorphisms.

EXAMPLE 2.10. If $V$ is the canonical complex line bundle over the complex projective 2-space, then $\operatorname{cd}(V ; K)=1, \operatorname{cd}(V ; K O)=2, \operatorname{cd}^{K}(V)=6$, and $\operatorname{cd}^{K O}(V)=\operatorname{cd}(V)=12$. See [12].

\section{§3. Elementary properties of codegrees}

THEOREM 3.1. Let $V$ be an orientable vector bundle over a connected finite $C W$ complex $X$. Then the following conditions are equivalent.

(1) $\operatorname{cd}_{p}(V)=0$.

(2) The order of $J(V)$ is prime to $p$, where $J(V)$ is the element represented by $V$ in the $J$-group $J(X)$.

(3) $\operatorname{cd}_{p}^{j}(V)=0$.

(4) $\operatorname{cd}_{p}^{j^{\prime}}(V)=0$. 
This is well-known. $\quad(1) \Leftrightarrow(2)$ is a corollary to a result of Kahn [10] (cf. Tanaka [15] and Dibag [8]); $(1) \Rightarrow(3) \Rightarrow(4)$ are trivial by $3.3(1)$ given below; $(4) \Rightarrow(2)$ is a consequence of results of Adams [2] in view of the fact (provided $p=2)$ that if $\operatorname{cd}_{2}^{j^{\prime}}(V)=0$ then $V$ has a spin structure. The last assertion is due to Knapp. Note that $\operatorname{cd}(V)=\# J(V)$, the order of $J(V)$, provided that $X$ is a double suspension (see e.g., [15]).

Theorem 3.2 (Crabb [4; p. 118]). Let $f: X \rightarrow Y$ be a Hurewicz fibration between connected finite $C W$ complexes such that each fibre has a homotopy type of a finite $C W$ complex. Let $V$ be an orientable vector bundle over $Y$. Then

$$
\operatorname{cd}\left(f^{*} V\right)|\operatorname{cd}(V)| \chi \operatorname{cd}\left(f^{*} V\right)
$$

where $\chi$ is the Euler characteristic of a fibre of $f$.

The first divisibility equation is trivial by definition. The second one is a consequence of the transfer $\tau: T(V) \rightarrow T\left(f^{*} V\right)$ of Becker-Gottlieb [3].

THEOREM 3.3. Let $V$ be an orientable vector bundle over a connected finite $C W$-complex $X$. Then we have

(1) $\operatorname{cd}_{p}^{K F}(V) \leqq \operatorname{cd}_{p}^{j F^{\prime}}(V) \leqq \operatorname{cd}_{p}^{j F}(V) \leqq \mathrm{cd}_{p}(V)$.

(2) $\operatorname{cd}_{p}^{j C}(V)=\operatorname{cd}_{p}^{j C^{\prime}}(V) \leqq \mathrm{cd}_{p}^{j^{\prime}}(V)$.

(3) $p \neq 2$.

(i ) $\operatorname{cd}_{p}^{K}(V)=\operatorname{cd}_{p}^{K o}(V)$.

(ii) $\operatorname{cd}_{p}^{j C^{\prime}}(V)=\operatorname{cd}_{p}^{j C}(V)=\operatorname{cd}_{p}^{j^{\prime}}(V)=\operatorname{cd}_{p}^{j}(V)$.

(iii) If $H_{*}(X ; Z)$ has no p-torsion, then $\operatorname{cd}_{p}^{K o}(V)=\operatorname{cd}_{p}^{j}(V)$.

(4) $p=2$.

(iv) $\operatorname{cd}_{2}^{K}(V) \leqq \mathrm{cd}_{2}^{K o}(V) \leqq 1+\operatorname{cd}_{2}^{K}(V)$.

(v) If $H_{*}(X ; Z)$ has no 2-torsion, then $\operatorname{cd}_{2}^{K}(V)=\mathrm{cd}_{2}^{j C}(V)$ and $\operatorname{cd}_{2}^{j^{\prime}}(V)$ $\leqq 1+\operatorname{cd}_{2}^{K o}(V)$.

(vi) If $H_{*}(X ; Z)$ and $K O^{n}(T(V))$ have no 2-torsion with $n=\operatorname{dim} V$, then $\operatorname{cd}_{2}^{K o}(V)=\operatorname{cd}_{2}^{j^{\prime}}(V)$.

Proof. Let $V$ be an $n$-dimensional orientable vector bundle over a connected finite $\mathrm{CW}$-complex $X$. We then have the following commutative diagram

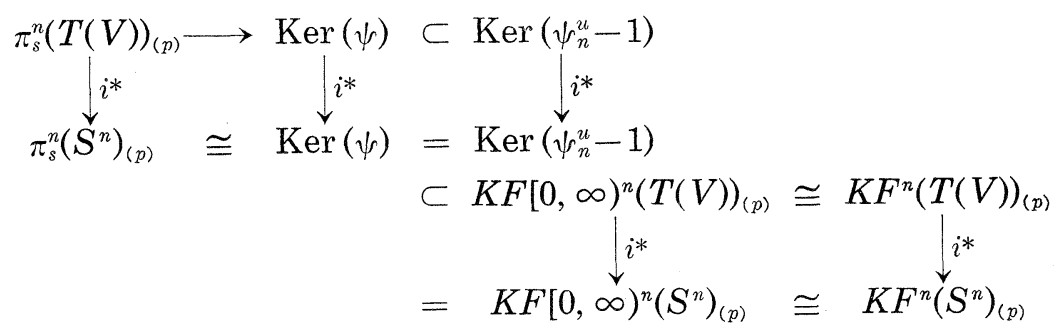


where the compositions of horizontal maps are the Hurewicz homomorphisms and $i: S^{n}=T\left(\left.V\right|_{x}\right) \subset T(V)$ is the inclusion. The exponents of $p$ in the orders of the cokernels of $i^{*}$ are, from the left, $\operatorname{cd}_{p}(V), \operatorname{cd}_{p}^{j F^{\prime}}(V)$ and $\mathrm{cd}_{p}^{j F^{\prime}}(V)$, respectively. Thus we have

$$
\operatorname{cd}_{p}^{j F^{\prime}}(V) \leqq \mathrm{cd}_{p}^{j F^{\prime}}(V) \leqq \mathrm{cd}_{p}(V) .
$$

From now on we assume $n \equiv 0(\bmod 8)$ by adding a trivial bundle to $V$. Consider the following commutative diagram.

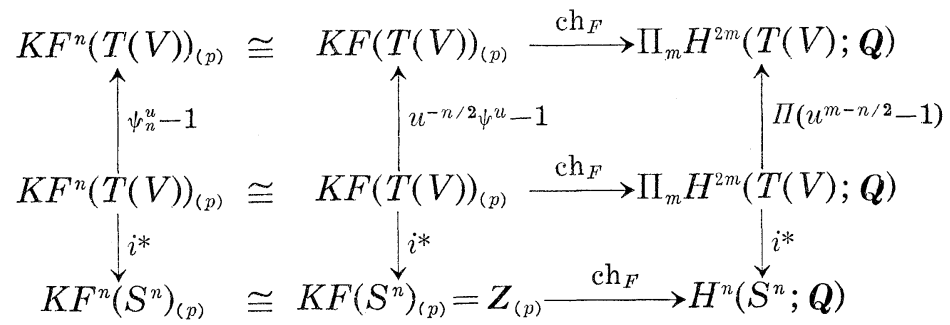

Set $e=\operatorname{cd}_{p}^{j F^{\prime}}(V)$. Then there exists $x \in \operatorname{Ker}\left(u^{-n / 2} \psi^{u}-1\right)$ with $i^{*}(x)=p^{e}$. Applying $\operatorname{ch}_{F}^{2 m}$, the $2 m$ dimensional component of $\operatorname{ch}_{F}$, to $u^{-n / 2} \psi^{u}(x)=x$, we have $u^{m-n / 2} \operatorname{ch}_{F}^{2 m}(x)=\operatorname{ch}_{F}^{2 m}(x)$ and hence $\left(u^{m-n / 2}-1\right) \operatorname{ch}_{F}^{2 m}(x)=0$. Therefore $\operatorname{ch}_{F}(x)=$ $\operatorname{ch}_{F}^{n}(x)$. We have $i^{*}\left( \pm p^{e} U_{H}(V)\right)= \pm p^{e} i^{*} U_{H}(V)= \pm p^{e} U_{H}\left(\left.V\right|_{x}\right)=\operatorname{ch}_{F} i^{*}(x)=$ $i^{*} \operatorname{ch}_{F}(x)=i^{*} \operatorname{ch}_{F}^{n}(x)$ so that $\pm p^{e} U_{H}(V)=\operatorname{ch}_{F}^{n}(x)=\operatorname{ch}_{F^{\prime}}(x)$ and $\operatorname{cd}_{p}^{K F}(V) \leqq e$. This completes the proof of (1).

We have a fibration $j \boldsymbol{C}_{n} \rightarrow j \boldsymbol{C}_{n}^{\prime} \rightarrow \mathscr{K}\left(\boldsymbol{Z}_{(p)}, n-1\right)$ from which we have $\operatorname{cd}_{p}^{j C}(V)=\operatorname{cd}_{p}^{j C^{\prime}}(V)$. By the complexification $c: j_{n}^{\prime} \rightarrow j C_{n}^{\prime}$, we also have $\operatorname{cd}_{p}^{j C^{\prime}}(V)$ $\leqq \mathrm{cd}_{p}^{j^{\prime}}(V)$. This proves (2).

By the complexification $c$, (i) is trivial. If $p$ is odd, then $c$ gives a homotopy equivalence $j_{n} \simeq j C_{n}^{\prime}$ and (ii) follows easily. Suppose that $H_{*}(X ; Z)$ is $p$-torsion free. Choose $y \in K F(T(V))$ with $\operatorname{ch}_{F}(y)=\operatorname{cd}^{K F}(V) U_{H}(V)$. Then $i^{*}(y)$ is $\operatorname{cd}^{K F}(V)$ times a generator of $K F\left(S^{n}\right)$ and $\operatorname{ch}_{F}^{2 m}(y)=0$ for $2 m>n$ so that $\operatorname{ch}_{F}\left(u^{-n / 2} \psi^{u}(y)\right)=\operatorname{ch}_{F}(y)$. Note that $\operatorname{ch}: K(T(V))_{(p)} \rightarrow H^{*}(T(V) ; \boldsymbol{Q})$ is injective by the assumption, and that $c: K O(T(V))_{(p)} \rightarrow K(T(V))_{(p)}$ is injective if $p$ is odd. It follows that if $F=C$ or $p$ is odd then $u^{-n / 2} \psi^{u}(y)=y$ and $\operatorname{socd}_{p}^{K F}(V)$ $\geqq \operatorname{cd}_{p}^{j F^{\prime}}(V)$ and hence $\operatorname{cd}_{p}^{K F^{\prime}}(V)=\mathrm{cd}_{p}^{j F^{\prime}}(V)$. This proves (iii) and a first half of (v). If $F=\boldsymbol{R}$ and $p=2$, then $\operatorname{ch} c\left(u^{-n / 2} \psi^{u}-1\right)=u^{-n / 2} \operatorname{ch} \psi^{u} c(y)-\operatorname{ch} c(y)=$ $u^{-n / 2} \psi_{H}^{u} \operatorname{ch}_{R}(y)-\operatorname{ch}_{R}(y)=0$ and so $c\left(u^{-n / 2} \psi^{u}-1\right)(y)=0$ and hence $\left(u^{-n / 2} \psi^{u}-1\right)$ $(2 y)=r c\left(u^{-n / 2} \psi^{u}-1\right)(y)=0$. Here $\psi_{H}^{u}: H^{2 k}(; \boldsymbol{Q}) \rightarrow H^{2 k}(; \boldsymbol{Q})$ is multiplication by $u^{k}$ and $r: K() \rightarrow K O()$ is the real restriction. Since $\operatorname{ch}_{R} i^{*}(2 y)=$ $i^{*} \operatorname{ch}_{R}(2 y)=2 \operatorname{cd}^{K o}(V) U_{H}\left(\left.V\right|_{x}\right)$ and $\operatorname{ch}_{R}: K O\left(S^{2 n}\right) \cong H^{2 n}\left(S^{2 n} ; Z\right)$, we have $\operatorname{cd}_{2}^{j^{\prime}}(V) \leqq 1+\operatorname{cd}_{2}^{K O}(V)$. This proves the second half of $(\mathrm{v})$. If moreover $K O^{n}(T(V))$ is 2-torsion free, then $c: K O(T(V))_{(2)} \rightarrow K(T(V))_{(2)}$ is injective and 
so $\left(u^{-n / 2} \psi^{u}-1\right)(y)=0$ and hence $\operatorname{cd}_{2}^{j^{\prime}}(V) \leqq \operatorname{cd}_{2}^{K o}(V)$. Thus $\operatorname{cd}_{2}^{j^{\prime}}(V)=\operatorname{cd}_{2}^{K o}(V)$ by (1). This proves (vi).

Finally we prove (iv) by showing

$$
\operatorname{cd}^{K}(V)\left|\operatorname{cd}^{K O}(V)\right| 2 \operatorname{cd}^{K}(V) .
$$

By the complexification $c$, the first part is trivial. Let $y \in K(T(V))$ with $\operatorname{ch}(y)=\operatorname{cd}^{K}(V) U_{H}(V)$. Then $\operatorname{ch}_{R}(r(y))=\operatorname{ch}\left(y+y^{*}\right)=2 \operatorname{ch}(y)=2 \operatorname{cd}^{K}(V) U_{H}(V)$, since $\operatorname{ch}\left(y^{*}\right)=\operatorname{ch} \psi^{-1} y=\psi_{H}^{-1} \operatorname{ch}(y)=(-1)^{n / 2} \operatorname{ch}(y)=\operatorname{ch}(y)$. Thus the second part follows. Here $y^{*}$ denotes the conjugate of $y$. This completes the proof of Theorem 3.3.

REMARK 3.4. Let $V$ be a multiple of the Hopf bundle over the complex or quaternionic projective spaces. Walker [17] and Ōshima [13] computed $\mathrm{cd}^{K O}(V)$. Crabb and Knapp [6], [7] computed $\mathrm{cd}^{j}(V)$ and gave an example $V$ such that $\operatorname{cd}^{K O}(V) \neq \operatorname{cd}^{j}(V)$.

ExAMPLE 3.5 (Crabb-Knapp [6]). If $V$ is a vector bundle over the real projective space, then $\operatorname{cd}^{j^{\prime}}(V)=\operatorname{cd}^{j}(V)=\operatorname{cd}(V)$.

Proof. Let $P\left(\boldsymbol{R}^{k}\right)$ and $L=L_{k}$ be the $(k-1)$-dimensional real projective space and the canonical line bundle over it, respectively. Since every vector bundle over $P\left(\boldsymbol{R}^{k}\right)$ is stably isomorphic to a multiple of $L$, it suffices to prove the case $V=n L$. If $n$ is odd, then $n L$ is non-orientable and so $\mathrm{cd}^{j^{\prime}}(n L)$ $=\operatorname{cd}^{j}(n L)=\mathrm{cd}(n L)=0$, by $2.5(5)$. Suppose $n$ is even. Then $n L$ is orientable. Let $\pi: S^{k-1} \rightarrow P\left(R^{k}\right)$ be the natural double covering. Then $\pi^{*} L$ is trivial and so $\mathrm{cd}(n L) \mid 2$ by 3.2 , hence $\mathrm{cd}^{j^{\prime}}(n L)=\mathrm{cd}^{j}(n L)=\mathrm{cd}(n L)$, by 3.1. Recall from [1] that $\# J(n L)=2^{f(k)} /\left(n, 2^{f(k)}\right)$, where $f(k)$ is the number of integers $s$ such that $0<s<k$ and $s \equiv 0,1,2,4(\bmod 8)$, and $(a, b)$ is the greatest common divisor of $a$ and $b$. Thus $\operatorname{cd}(n L)$ is 0 if $n$ is odd, 1 if $n \equiv 0\left(\bmod 2^{f(k)}\right)$ and 2 otherwise.

REMARK 3.6. There is a computational proof of 3.5 and it shows that $\operatorname{cd}^{K F}\left(n L_{k}\right) \neq \operatorname{cd}^{j F}\left(n L_{k}\right)$ for some $n, k$.

\section{§4. Proof of Theorem 1.1}

We denote by $\mathscr{K}(A, m)$ an $\Omega$-spectrum whose $i$-th term is the Eilenberg-MacLane complex $K(A, m+i)$ for $m+i \geqq 1$.

We have fibrations of $\Omega$-spectra:

$$
\begin{gathered}
K O[1, \infty) \stackrel{b_{0}}{\longrightarrow} K O[0, \infty) \stackrel{k_{0}}{\longrightarrow} \mathscr{K}(Z, 0), \\
\mathscr{K}\left(Z_{2}, 0\right) \stackrel{j_{2}}{\longrightarrow} K O[2, \infty) \stackrel{b_{1}}{\longrightarrow} K O[1, \infty) \stackrel{k_{1}}{\longrightarrow} \mathscr{K}\left(Z_{2}, 1\right),
\end{gathered}
$$




$$
\begin{gathered}
\mathscr{K}\left(Z_{2}, 1\right) \stackrel{j_{4}}{\longrightarrow} K O[4, \infty) \stackrel{b_{2}}{\longrightarrow} K O[2, \infty) \stackrel{k_{2}}{\longrightarrow} \mathscr{K}\left(Z_{2}, 2\right), \\
K O[4, \infty) \stackrel{\gamma}{\longrightarrow} K O[0, \infty) \stackrel{\beta}{\longrightarrow} K O[0,2]
\end{gathered}
$$

where $\gamma=b_{0} \circ b_{1} \circ b_{2}$ and $K O[0,2]$ is a delooping of the fibre spectrum of $\gamma$ such that

$$
\begin{array}{ll}
\beta_{*}: \pi_{m}(K O[0, \infty)) \cong \pi_{m}(K O[0,2]), & m \leqq 2, \\
\pi_{m}(K O[0,2])=0, & m \geqq 3 .
\end{array}
$$

LEMMA 4.5 (Thomas $[16 ; 4.2]) . \quad k_{2} \circ j_{2}=S q^{2}$.

The next lemma can be proved easily, so we omit its proof.

LEMMA 4.6. Let $E$ be an $\Omega$-spectrum and let $Y$ be a connected $C W$ complex. Then the canonical homomorphism $E[k, \infty)^{m}(Y) \rightarrow E^{m}(Y)$ is a monomorphism or an isomorphism if $Y$ is $(m+k-2)$-connected or $(m+k-1)$-connected, respectively.

For any CW complex $Y$ and any vector bundle $V$ over $Y$, we denote by $Y^{m}$ the $m$-skeleton of $Y$ and we give $T(V)$ a cell structure naturally so that $T(V)^{m+n}=T\left(V \mid Y^{m}\right)$, where $n=\operatorname{dim} V$.

LEMma 4.7. Let $X$ be a connected finite $C W$ complex with a single vertex and let $V$ be an $n$-dimensional orientable vector bundle over $X$ with $n \geqq 1$. Set $T=T(V)$. Then we have

(1) The following groups are isomorphic with each other.

$$
\begin{aligned}
& A_{1}=\operatorname{Ker}\left[\gamma_{*}: K O[4, \infty)^{n}(T) \longrightarrow K O[0, \infty)^{n}(T)\right], \\
& A_{2}=\operatorname{Coker}\left[\beta_{*}: K O[0, \infty)^{n-1}(T) \longrightarrow K O[0,2]^{n-1}(T)\right], \\
& A_{3}=\operatorname{Coker}\left[i^{*}: K O[0, \infty)^{n-1}(T) \longrightarrow K O[0, \infty)^{n-1}\left(T^{n+2}\right)\right], \\
& A_{4}=\operatorname{Coker}\left[i^{*}: K O^{n-1}(T) \longrightarrow K O^{n-1}\left(T^{n+2}\right)\right], \\
& A_{5}=\operatorname{Coker}\left[i^{*}: K O[1, \infty)^{n-1}(T) \longrightarrow K O[1, \infty)^{n-1}\left(T^{n+2}\right)\right], \\
& A_{6}=\operatorname{Coker}\left[i^{*}: K O[2, \infty)^{n-1}(T) \longrightarrow K O[2, \infty)^{n-1}\left(T^{n+2}\right)\right], \\
& A_{7}=\operatorname{Coker}\left[k_{2 *}: K O[2, \infty)^{n-1}(T) \longrightarrow H^{n+1}\left(T ; Z_{2}\right)\right], \\
& A_{8}=\operatorname{Coker}\left[k_{2 *}: K O[2, \infty)^{n-1}\left(T / T^{n}\right) \longrightarrow H^{n+1}\left(T / T^{n} ; Z_{2}\right)\right], \\
& A_{9}=\operatorname{Coker}\left[i^{*}: K O[2, \infty)^{n-1}\left(T / T^{n}\right) \longrightarrow K O[2, \infty)^{n-1}\left(T^{n+2} / T^{n}\right)\right], \\
& A_{10}=\operatorname{Im}\left[j_{4 *}: H^{n+1}\left(T ; Z_{2}\right) \longrightarrow K O[4, \infty)^{n}(T)\right], \\
& A_{11}=\operatorname{Ker}\left[\gamma_{*}: K O[4, \infty)^{n}(T)_{(2)} \longrightarrow K O[0, \infty)^{n}(T)_{(2)}\right],
\end{aligned}
$$

where Coker means the cokernel. In particular $A_{11}$ is a 2-group, that is, $2 a=0$ for every $a \in A_{11}$. 
(2) If $H^{1}\left(X ; Z_{2}\right)=0$ or $w_{2}(V)=0$, then all $A_{i}$ in (1) are trivial groups.

(3) If $X$ has only one 1-cell and $H^{1}\left(X ; Z_{2}\right)=Z_{2}$, then all $A_{i}$ in (1) are isomorphic to

$$
A_{12}=\operatorname{Coker}\left[i^{*}: K O^{n-1}\left(T / T^{n}\right) \longrightarrow K O^{n-1}\left(T^{n+1} / T^{n}\right)\right] .
$$

Proof. For simplicity, we denote by $H^{*}(\quad)$ the ordinary cohomology theory with $Z_{2}$-coefficients.

By (4.4), we have $A_{1} \cong A_{2}$ and

$$
\beta_{*}: K O[0, \infty)^{n-1}\left(T^{n+2}\right) \cong K O[0,2]^{n-1}\left(T^{n+2}\right) .
$$

Since $K O[0,2]_{n-1}$ and $K O[0,2]_{n}$ are $(n+3)$-coconnected, it follows that $K O[0,2]^{n-1}\left(T / T^{n+2}\right)$ and $K O[0,2]^{n}\left(T / T^{n+2}\right)$ are trivial groups, so the lower $i^{*}$ in the following commutative square is an isomorphism, hence $A_{2} \cong A_{3}$.

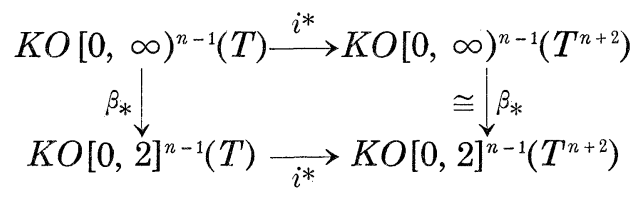

By 4.6, we have $A_{3} \cong A_{4} \cong A_{5}$.

Since $b_{1 *}: K O[2, \infty)^{n}\left(T / T^{n+2}\right) \cong K O[1, \infty)^{n}\left(T / T^{n+2}\right)$ by $4.6, b_{1}$ induces an injection

$$
A_{6} \subset A_{5}
$$

We can show that this injection is an isomorphism and that if $w_{2}(V)=0$ then $A_{6}=A_{5}=0$ in the following way.

As is well-known, we have

$$
\phi^{-1} S q^{2} \phi(1)=w_{2}(V) \in H^{2}(X)
$$

where $\phi: H^{*}\left(X^{+}\right) \rightarrow H^{*+n}(T)$ is the Thom isomorphism. Here $X^{+}$is the union of $X$ and the disjoint base point. Notice from 4.5 that $k_{2 *} \circ j_{2 *}=S q^{2}$ on $H^{n}(T)$ and $H^{n}\left(T^{n+2}\right)$.

If $w_{2}(V) \neq 0$, then $j_{2 *}$ is injective on $H^{n}\left(T^{n+2}\right)$, so $b_{1 *}$ is surjective and hence isomorphic by $(4.2)$ on $K O[2, \infty)^{n-1}\left(T^{n+2}\right)$. Thus we have $A_{6} \cong A_{5}$.

If $w_{2}(V)=0$, then $V$ is $K O$-orientable, so the Thom isomorphism induces an isomorphism

$$
A_{4} \cong \operatorname{Coker}\left[i^{*}: K O^{-1}(X) \longrightarrow K O^{-1}\left(X^{2}\right)\right] .
$$

By 4.6, the last group is isomorphic to

$$
A_{4}^{\prime}=\operatorname{Coker}\left[i^{*}: K O[2, \infty)^{-1}(X) \longrightarrow K O[2, \infty)^{-1}\left(X^{2}\right)\right] .
$$


By the cofibration $X^{1} \rightarrow X^{2} \rightarrow X^{2} / X^{1}$ and the five lemma, we can show that the right $k_{2 *}$ in the following commutative diagram is an isomorphism; hence $A_{4}^{\prime}$ is isomorphic to the cokernel of the left $k_{2 *}$.

$$
\begin{gathered}
{[X, S O]=K O[2, \infty)^{-1}(X) \stackrel{i^{*}}{\longrightarrow} K O[2, \infty)^{-1}\left(X^{2}\right)} \\
k_{2 *} \downarrow \\
{\left[X, K\left(Z_{2}, 1\right)\right]=H^{1}(X) \cong k_{2 *}}
\end{gathered}
$$

Since there exists an embedding i: $P\left(\boldsymbol{R}^{\infty}\right) \subset S O$ such that $k_{2} \circ i: P\left(\boldsymbol{R}^{\infty}\right) \rightarrow$ $K\left(Z_{2}, 1\right)$ is a homotopy equivalence [18], it follows that the left $k_{2 *}$ in the diagram above is surjective, so $A_{4}^{\prime}=0$, hence $A_{6}=A_{5}=0$.

Since $K O[4, \infty)^{n-1}\left(T^{n+2}\right)$ and $K O[4, \infty)^{n}\left(T^{n+2}\right)$ are trivial groups, the lower $k_{2 *}$ in the following commutative square is an isomorphism.

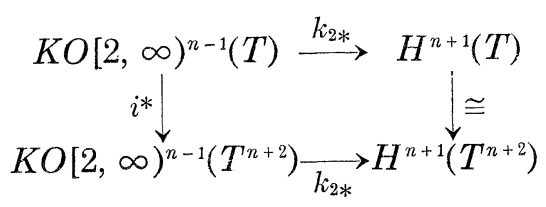

Hence we have $A_{6} \cong A_{7}$.

Consider the following commutative diagram, where $q: T \rightarrow T / T^{n}$ is the quotient map.

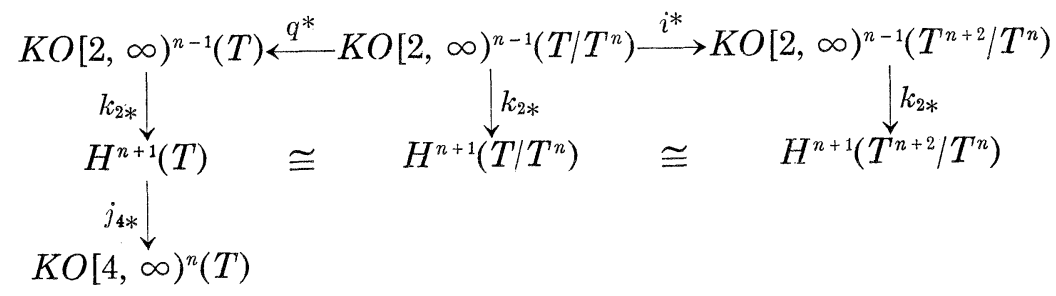

Since $K O[2, \infty)^{n-1}\left(T^{n}\right)=0, q^{*}$ is a surjection. By the exact sequences of the triple $\left(T^{n+2}, T^{n+1}, T^{n}\right)$, the third $k_{2 *}$ is an isomorphism. Hence we have $A_{7} \cong$ $A_{8} \cong A_{9} \cong A_{10}$.

Since $A_{7}$ is a 2 -group and since localization preserves exactness, it follows that $A_{1}$ is a 2 -group and $A_{1} \cong\left(A_{1}\right)_{(2)} \cong A_{11}$. Since $H^{n+1}(T) \cong H^{1}(X)$, this completes the proofs of (1) and (2).

To prove (3), suppose that $X$ has a single 1-cell and $H^{1}(X)=Z_{2}$. By the commutative diagram

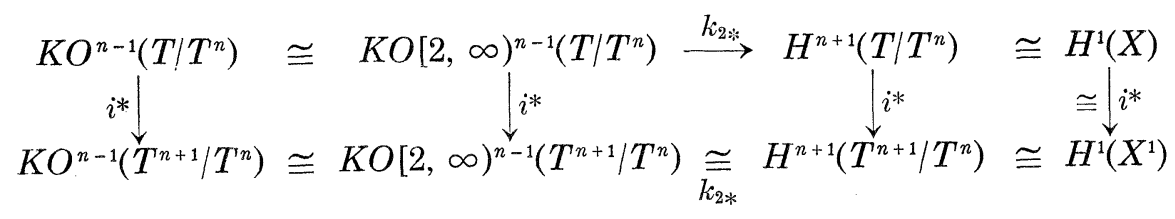


we have $A_{8} \cong A_{12}$. Hence (3) follows from (1). Note that the fourth $i^{*}$ is an isomorphism by the assumption on $H^{1}(X)$. This completes the proof of 4.7 .

Now we prove Theorem 1.1. We may assume that $V$ is orientable by 2.5(5) and that $X$ has a single vertex. Let $p=2$ and let $y \in \operatorname{Ker}\left(\psi_{*}^{u}-1\right) \subset$ $K O[0, \infty)^{n}(T(V))_{(2)}$ with $i^{*}(y)=2^{e}$, where $n=\operatorname{dim} V$ and $e=\operatorname{cd}_{2}^{j^{\prime}}(V)$. Then $\gamma_{*} \psi(y)=\left(\psi_{*}^{u}-1\right)(y)=0$, so $\psi(y) \in \operatorname{Ker}\left(\gamma_{*}\right)$. It follows from 4.7(1) that $2 \psi(y)$ $=0$, so $2 y \in \operatorname{Ker}(\psi)$ with $i^{*}(2 y)=2^{1+e}$, hence $\operatorname{cd}_{2}^{j}(V) \leqq 1+\operatorname{cd}_{2}^{j^{\prime}}(V)$ and $\operatorname{cd}^{j}(V) \mid$ $2 \mathrm{~cd}^{j^{\prime}}(V)$ by $3.3(\mathrm{ii})$. If $H^{1}\left(X ; Z_{2}\right)=0$ or $w_{2}(V)=0$, then $\gamma_{*}: K O[4, \infty)^{n}(T(V))_{(2)}$ $\rightarrow K O[0, \infty)^{n}(T(V))_{(2)}$ is injective by 4.7 , $\operatorname{so~}_{\operatorname{cd}_{2}^{j}}(V)=\operatorname{cd}_{2}^{j^{\prime}}(V)$ and $\operatorname{cd}^{j}(V)=$ $\mathrm{cd}^{j^{\prime}}(V)$. This completes the proof of Theorem 1.1.

\section{§5. Proof of Theorem 1.2}

We work in the stable homotopy category of CW complexes. Let $\eta$ : $S^{1} \rightarrow S^{0}$ be the generator of the stable 1-stem which is of order 2 .

Let $m \geqq 2$ and let $\pi: L^{\infty}(m)=S^{\infty} / Z_{m} \rightarrow P_{\infty}=S^{\infty} / S^{1}$ be the projection from the standard lens space to the complex projective space. As is well-known, $L^{\infty}(m)$ and $P_{\infty}$ have cell structures

$$
\begin{aligned}
& L^{\infty}(m)=\bigcup_{n \geqq 0} e^{n}, \\
& P_{\infty}=\bigcup_{n \geqq 0} e^{2 n}
\end{aligned}
$$

such that $\pi$ induces a homeomorphism of $S^{2 n}$ for each $n$ :

$$
\pi: L^{2 n}(m) / L^{2 n-1}(m)=P_{n+1} / P_{n},
$$

where $L^{k}(m)$ is the $k$-skeleton of $L^{\infty}(m)$ and $P_{k}$ is the $(2 k-2)$-skeleton of $P_{\infty}$. We denote the restriction $L^{2 k-1}(m) \rightarrow P_{k}$ of $\pi$ by also $\pi$. Let $H_{k}$ be the canonical complex line bundle over $P_{k}$. Then there are natural homeomorphisms

$$
\begin{aligned}
& T\left(n \pi^{*} H_{k+1}\right)=L^{2 n+2 k+1}(m) L^{2 n-1}(m), \\
& T\left(n \pi^{*} H_{k+1} \mid e^{c}\right)=L^{2 n}(m) / L^{2 n-1}(m)=S^{2 n} .
\end{aligned}
$$

We have also

$$
\begin{gathered}
H^{*}\left(L^{2 k}(m) ; \boldsymbol{Q}\right)=0, \\
L^{2 n+3}(m) / L^{2 n+1}(m)=S^{2 n+2} \bigcup_{0} e^{2 n+3}=S^{2 n+2} \vee S^{2 n+3}, \\
L^{2 n+2}(m) / L^{2 n}(m)=S^{2 n+1} \bigcup_{m} e^{2 n+2},
\end{gathered}
$$

where the attaching map $r: S^{t} \rightarrow S^{t}$ means a map of degree $r$.

Lemma 5.3. Let $m \equiv 0(\bmod 4)$. Then we have

(1) $H^{*}\left(L^{2 k+1}(m)^{+} ; Z_{2}\right)=\bigwedge_{2}(x) \otimes Z_{2}[y] /\left(y^{k+1}\right)$, where $\bigwedge_{2}$ is an exterior algebra over $Z_{2}, \operatorname{dim} x=1$ and $\operatorname{dim} y=2$. 
(2) $Z_{2}[y] /\left(y^{k+1}\right)=\operatorname{Im}\left[\pi^{*}: H^{*}\left(P_{k+1}^{+} ; Z_{2}\right) \rightarrow H^{*}\left(L^{2 k+1}(m)^{+} ; Z_{2}\right)\right]$.

(3) $L^{2 n+2}(m) / L^{2 n-1}(m)=\left(S^{2 n} \vee S^{2 n+1}\right) \bigcup_{f} e^{2 n+2}$, where $f=n \eta \vee m: S^{2 n+1} \rightarrow$ $S^{2 n} \vee S^{2 n+1}$.

(4) $L^{2 n+3}(m) / L^{2 n-1}(m)=\left(S^{2 n} \bigvee S^{2 n+1}\right) \bigcup_{f} e^{2 n+2} \bigcup_{g} e^{2 n+3}$, where g is a map $S^{2 n+2}$ $\rightarrow S^{2 n} \vee S^{2 n+1}$ having the second factor $n \eta$.

Proof. (1) and (2) are well-known. Since $S q^{2}\left(x y^{n}\right)=n\left(x y^{n+1}\right)$ by (1) and (2), the first factor of $f$ and the second factor of $g$ are $n \eta$. The second factor of $f$ is $m$, by (5.2).

THEOREM 5.4. Let $V$ be a vector bundle over $L^{4}(m)$. Then

(1) $\operatorname{cd}^{j}(V)=\mathrm{cd}(V)$ and $\mathrm{cd}_{p}^{j^{\prime}}(V)=\operatorname{cd}_{p}^{j}(V)$ for every odd prime $p$.

(2) If $\nu_{2}(m) \leqq 1$, then $\operatorname{cd}_{2}^{j^{\prime}}(V)=\operatorname{cd}_{2}^{j}(V)$ and hence $\operatorname{cd}^{j^{\prime}}(V)=\operatorname{cd}^{j}(V)$.

Proof. By 2.5(5), it suffices to consider the case that $V$ is orientable. We have (1) by 2.9 and 3.3(3). As is easily proved, $K O\left(L^{4}(m)\right)$ is finite, hence the inclusion induces an isomorphism $K O\left(L^{5}(m)\right) \cong K O\left(L^{4}(m)\right)$. Therefore every vector bundle over $L^{4}(m)$ can be extended stably to $L^{5}(m)$. On the other hand every vector bundle over $S^{5}$ is stably trivial. Thus every orientable vector bundle $V$ over $L^{4}(m)$ satisfies cd $(V) \mid m$, by 3.2. This and 3.1 imply (2).

Set $H_{4}^{\prime}=(\pi i) * H_{3}$, where

$$
L^{4}(m) \stackrel{i}{\subset} L^{5}(m) \stackrel{\pi}{\longrightarrow} P_{3} .
$$

Since $w_{2}\left(n H_{4}^{\prime}\right)=0$ for $n$ even, (1) of the next theorem follows from Theorem 1.1, and Theorem 1.2 is a corollary to 5.4 and $5.5(2)$.

THEOREM 5.5. (1) If $n$ is even, then $\operatorname{cd}^{j^{\prime}}\left(n H_{4}^{\prime}\right)=\operatorname{cd}^{j}\left(n H_{4}^{\prime}\right)$.

(2) If $m \equiv 0$ (4) and $n$ is odd, then $\mathrm{cd}^{K O}\left(n H_{4}^{\prime}\right)=2$ and we have the following table in which the first entry is $\operatorname{cd}_{2}^{j^{\prime}}\left(n H_{4}^{\prime}\right)$ and the second is $\mathrm{cd}_{2}^{j}\left(n \mathrm{H}_{4}^{\prime}\right)$.

\begin{tabular}{|c|c|c|c|}
\hline \multicolumn{1}{|c|}{$\nu_{2}(m)$} & 2 & 3 & $4 \leqq$ \\
\hline 1 & & & \\
\hline 3,7 & 1,1 & 1,2 & 2,2 \\
\hline 5 & 1,2 & 2,3 & 3,3 \\
\hline 1,1 & 1,1 & 1,1 \\
\hline
\end{tabular}

REMARK 5.6. By 3.2, we have $\operatorname{cd}\left(n H_{4}^{\prime}\right) \mid \operatorname{cd}\left(n H_{3}\right)$, where $\operatorname{cd}\left(n H_{3}\right)=W^{s}\{a, 3\}$ with $n+a \equiv 0(24)$ and $W^{s}\{a, 3\}$ was completely determined [13], for example 


$$
\operatorname{cd}_{2}\left(n H_{3}\right)= \begin{cases}1 & \text { if } n \equiv 5(8) \\ 2 & \text { if } n \equiv 1(8) \\ 3 & \text { if } n \equiv 3(4)\end{cases}
$$

To prove 5.5(2) we must make some preparations. Set

$$
\begin{aligned}
& T=T\left(n H_{4}^{\prime}\right)=L^{2 n+4}(m) / L^{2 n-1}(m)=S^{2 n} \bigvee S^{2 n+1} \bigcup_{f} e^{2 n+2} \bigcup_{g} e^{2 n+3} \bigcup_{h} e^{2 n+4}, \\
& T_{1}=L^{2 n+3}(m) / L^{2 n-1}(m)=S^{2 n} \bigvee S^{2 n+1} \bigcup_{f} e^{2 n+2} \bigcup_{g} e^{2 n+3}, \\
& T_{0}=L^{2 n+2}(m) / L^{2 n-1}(m)=S^{2 n} \bigvee S^{2 n+1} \bigcup_{f} e^{2 n+2}, \\
& P_{n+k, k}=P_{n+k} / P_{n},
\end{aligned}
$$

where $f$ and $g$ are the maps in 5.3, and $h: S^{2 n+3} \rightarrow T_{1}$ is the attaching map of the top cell of $T$. Collapsing maps will be denoted by $q$. We denote the canonical maps $T \rightarrow P_{n+3,3}$ and $T_{1} \rightarrow P_{n+2,2}$ by $\pi$. Inclusions will be denoted by $i$.

From now on we consider only the case $p=2$ and $u \equiv 3(8)$. For an integer $n$ and rational numbers $a, b$, we define a rational number $n_{a, b}$ by

$$
n_{a, b}=\left(u^{2}-1\right)\{(1 / 24)(n+3) a+b\} \text {. }
$$

We shall prove

Proposition 5.7. Let $m \equiv 0$ (4) and $n \equiv 1(2)$. Then

(1) $\operatorname{Im}\left[i^{*}: K O^{2 n}(T) \rightarrow K O^{2 n}\left(S^{2 n}\right)\right]=2 K O^{2 n}\left(S^{2 n}\right)$, and there exist elements $z_{2}^{\prime \prime \prime}, z_{1}, z_{2}$ such that

(2) $K O[4, \infty)^{2 n}(T)=Z_{m}\left\{z_{2}^{\prime \prime \prime}\right\}$,

(3) $K O^{2 n}(T)=Z\left\{z_{1}\right\} \oplus Z_{m / 2}\left\{z_{2}\right\}$ and $z_{2}^{\prime \prime \prime}$ projects to $z_{2}$,

(4) $\left(\psi_{2 n}^{u}-1\right)\left(a z_{1}+b z_{2}\right)=n_{a, b} z_{2}$ and $\psi\left(a z_{1}+b z_{2}\right)=n_{a, b} z_{2}^{\prime \prime \prime}$.

Now we prove 5.5(2) by 5.7. Recall from 2.7 that the 2-components of $\mathrm{cd}^{j^{\prime}}\left(n H_{4}^{\prime}\right)$ and $\mathrm{cd}^{j}\left(n H_{4}^{\prime}\right)$ are the orders of the cokernels of the composites

$$
\begin{aligned}
& \operatorname{Ker}\left(\psi_{2 n}^{u}-1\right) \subset K O^{2 n}(T)_{(2)} \stackrel{i^{*}}{\longrightarrow} K O^{2 n}\left(S^{2 n}\right)_{(2)}, \\
& \operatorname{Ker}(\psi) \subset K O^{2 n}(T)_{(2)} \longrightarrow K O^{2 n}\left(S^{2 n}\right)_{(2)},
\end{aligned}
$$

respectively. Suppose $m \equiv 0(4)$ and $n$ is odd. By 5.7, $\operatorname{Im}\left(i^{*}\right)=2 K O^{2 n}\left(S^{2 n}\right)_{(2)}$ and

$$
\begin{aligned}
& a z_{1}+b z_{2} \in \operatorname{Ker}\left(\psi_{2 n}^{u}-1\right) \Longleftrightarrow \nu_{2}\left(n_{a, b}\right) \geqq \nu_{2}(m / 2), \\
& a z_{1}+b z_{2} \in \operatorname{Ker}(\psi) \Longleftrightarrow \nu_{2}\left(n_{a, b}\right) \geqq \nu_{2}(m) .
\end{aligned}
$$

Suppose $m \equiv 8(16)$ and $n \equiv 3(4)$. If $\nu_{2}\left(n_{a, b}\right) \geqq 2$, then $\nu_{2}(a) \geqq 1$. In fact $a=2$ and $b=-(n+1) / 12$ satisfy it. Hence $\operatorname{cd}_{2}^{j^{\prime}}\left(n H_{4}^{\prime}\right)=2$. If $\nu_{2}\left(n_{a, b}\right) \geqq 3$, then $\nu_{2}(a)$ $\geqq 2$. In fact $a=4$ and $b=-(n+1) / 6$ satisfy it. Hence $\operatorname{cd}_{2}^{j}\left(n H_{4}^{\prime}\right)=3$. 
By the same metbod, we can calculate all $\mathrm{cd}_{2}^{j^{\prime}}\left(n H_{4}^{\prime}\right)$ and $\operatorname{cd}_{2}^{j}\left(n H_{4}^{\prime}\right)$. We omit the details.

By the commutative diagram:

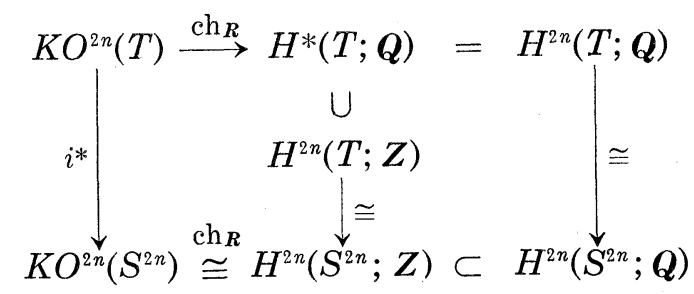

and 5.7(1), we have $\operatorname{cd}^{K o}\left(n H_{4}^{\prime}\right)=2$. This proves 5.5(2).

The rest of this section is devoted to the proof of 5.7.

LEMMA 5.8. Let $m \equiv 0(4)$ and $n \equiv 1(2)$. Then

(1) $K O[4, \infty)^{2 n}(T)=Z_{m}$ and $q^{*}: K O[4, \infty)^{2 n}\left(S^{2 n+4}\right) \rightarrow K O[4, \infty)^{2 n}(T)$ is surjective.

(2) $\operatorname{Ker}\left[K O[4, \infty)^{2 n}(T) \rightarrow K O[0, \infty)^{2 n}(T)\right]=Z_{2}$.

(3) $K O^{2 n-1}\left(T_{1}\right)=Z, K O^{2 n-1}\left(T_{0}\right)=Z_{2}$ and $\operatorname{Im}\left[q^{*}: K O^{2 n-1}\left(S^{2 n+3}\right) \rightarrow K O^{2 n-1}\left(T_{1}\right)\right]$ $=2 K O^{2 n-1}\left(T_{1}\right)$.

(4) $\operatorname{Im}\left[h^{*}: K O^{2 n-1}\left(T_{1}\right) \rightarrow K O^{2 n-1}\left(S^{2 n+3}\right)\right]=(m / 2) K O^{2 n-1}\left(S^{2 n+3}\right)$.

(5) $K O^{2 n}\left(T_{1}\right)=Z, K O^{2 n}\left(T_{0}\right)=Z \oplus Z_{2}$, the inclusions induce an isomorphism $K O^{2 n}\left(T_{1}\right) \cong K O^{2 n}\left(T_{0}\right) / Z_{2}$ and an epimorphism $K O^{2 n}(T) \rightarrow K O^{2 n}\left(T_{1}\right)$, and $\operatorname{Im}\left[i^{*}\right.$ : $\left.K O^{2 n}\left(T_{0}\right) \rightarrow K O^{2 n}\left(S^{2 n}\right)\right]=2 K O^{2 n}\left(S^{2 n}\right)$.

(6) $\pi^{*}: K O^{2 n}\left(P_{n+2,2}\right) \cong K O^{2 n}\left(T_{1}\right)$.

(7) We have the commutative diagram:

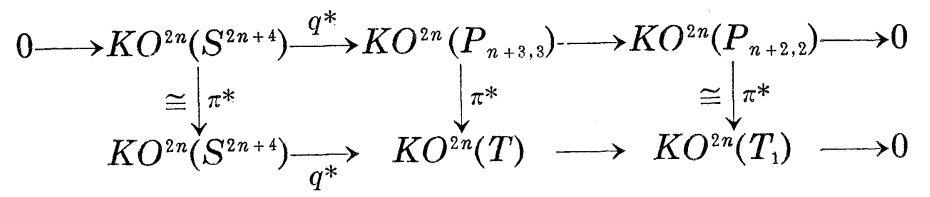

Here the second $\pi^{*}$ is surjective and the image of the lower $q^{*}$ is $Z_{m / 2}$.

(8) $\pi^{*}: K O[4, \infty)^{2 n}\left(P_{n+3,3}\right) \rightarrow K O[4, \infty)^{2 n}(T)$ is surjective.

Proof. We have $q^{*}: K O[4, \infty)^{2 n-1}\left(S^{2 n+3}\right) \cong K O[4, \infty)^{2 n-1}\left(T_{1}\right)$ by the exact sequence of $\left(T_{1}, T_{0}\right)$ and the relation $q \circ h=m$. The last relation and the exact sequence

$$
\begin{aligned}
K O[4, \infty)^{2 n-1}\left(T_{1}\right) & \stackrel{h^{*}}{\longrightarrow} K O[4, \infty)^{2 n-1}\left(S^{2 n+3}\right) \\
& \longrightarrow K O[4, \infty)^{2 n}(T) \longrightarrow K O[4, \infty)^{2 n}\left(T_{1}\right)=0
\end{aligned}
$$

proves (1). Note that (1) holds without the assumption on $m$ and $n$. 
Suppose $m \equiv 0(4)$ and $n \equiv 1(2)$.

By 4.7(3), $\operatorname{Ker}\left[K O[4, \infty)^{2 n}(T) \rightarrow K O[0, \infty)^{2 n}(T)\right] \cong \operatorname{Coker}\left[i^{*}: K O^{2 n-1}\left(T / S^{2 n}\right)\right.$ $\left.\rightarrow K O^{2 n-1}\left(S^{2 n+1}\right)\right]$. To prove (2), we calculate the last group. Since $T_{0} / S^{2 n}=$ $S^{2 n+1} \cup_{m} e^{2 n+2}$, it is easy to show that

$$
i^{*}: K O^{2 n-1}\left(T_{0} / S^{2 n}\right) \cong K O^{2 n-1}\left(S^{2 n+1}\right), \quad K O^{2 n-2}\left(T_{0} / S^{2 n}\right)=Z_{m} .
$$

By the last equations, we have an exact sequence:

$$
0 \longrightarrow K O^{2 n-1}\left(S^{2 n+3}\right) \stackrel{q^{*}}{\longrightarrow} K O^{2 n-1}\left(T_{1} / S^{2 n}\right) \stackrel{i^{*}}{\longrightarrow} K O^{2 n-1}\left(T_{0} / S^{2 n}\right)=Z_{2} \longrightarrow 0
$$

By 5.3(4), we have an exact sequence

$$
\begin{aligned}
0=K O^{2 n-1}\left(S^{2 n+2}\right) & \longrightarrow K O^{2 n-1}\left(T_{1} / S^{2 n}\right) \\
& \longrightarrow K O^{2 n-1}\left(S^{2 n+1} \bigcup_{\eta} e^{2 n+3}\right) \longrightarrow K O^{2 n}\left(S^{2 n+2}\right)=Z_{2} .
\end{aligned}
$$

Since $K O^{2 n-1}\left(S^{2 n+1} \bigcup_{\eta} e^{2 n+3}\right) \cong K O\left(P_{3}\right)=Z$, we have $K O^{2 n-1}\left(T_{1} / S^{2 n}\right) \cong Z$ and so

$$
\operatorname{Im}\left[q^{*}: K O^{2 n-1}\left(S^{2 n+3}\right) \longrightarrow K O^{2 n-1}\left(T_{1} / S^{2 n}\right)\right]=2 K O^{2 n-1}\left(T_{1} / S^{2 n}\right) .
$$

Let $h^{\prime}: S^{2 n+3} \rightarrow T_{1} / S^{2 n}$ be the attaching map of the top cell of $T / S^{2 n}$. Then $q \circ h^{\prime}=m$. Hence $h^{\prime *} q^{*}=m$ on $K O^{2 n-1}\left(S^{2 n+3}\right)$ and $\operatorname{Im}\left[h^{* *}: K O^{2 n-1}\left(T_{1} / S^{2 n}\right) \rightarrow\right.$ $\left.K O^{2 n-1}\left(S^{2 n+3}\right)\right]=(m / 2) K O^{2 n-1}\left(S^{2 n+3}\right)$ and in particular $h^{* *}$ is injective. Therefore by the exact sequence of $\left(T / S^{2 n}, T_{1} / S^{2 n}\right)$ we have $K O^{2 n-1}\left(T / S^{2 n}\right)=0$ and so

$$
\text { Coker }\left[i^{*}: K O^{2 n-1}\left(T / S^{2 n}\right) \longrightarrow K O^{2 n-1}\left(S^{2 n+1}\right)\right]=K O^{2 n-1}\left(S^{2 n+1}\right)=Z_{2} .
$$

This proves (2).

Consider the exact sequence

$$
K O^{2 n-1}\left(S^{2 n+3}\right) \stackrel{q^{*}}{\longrightarrow} K O^{2 n-1}\left(T_{1}\right) \longrightarrow K O^{2 n-1}\left(T_{0}\right) \longrightarrow K O^{2 n}\left(S^{2 n+3}\right)=0 .
$$

By $5.3(3)$, we can prove easily $K O^{2 n-1}\left(T_{0}\right)=Z_{2}$. Since $h^{*} q^{*}=m, K O^{2 n-1}\left(T_{1}\right)$ is $\boldsymbol{Z}$ or $\boldsymbol{Z} \oplus \boldsymbol{Z}_{2}$. If $K O^{2 n-1}\left(T_{1}\right)=\boldsymbol{Z} \oplus \boldsymbol{Z}_{2}$, then $\operatorname{Im}\left[h^{*}: K O^{2 n-1}\left(T_{1}\right) \rightarrow K O^{2 n-1}\left(S^{2 n+3}\right)\right]=$ $m K O^{2 n-1}\left(S^{2 n+3}\right)$, and so $\operatorname{Im}\left[q^{*}: K O^{2 n}\left(S^{2 n+4}\right) \rightarrow K O^{2 n}(T)\right]=Z_{m}$, hence by the commutative diagram

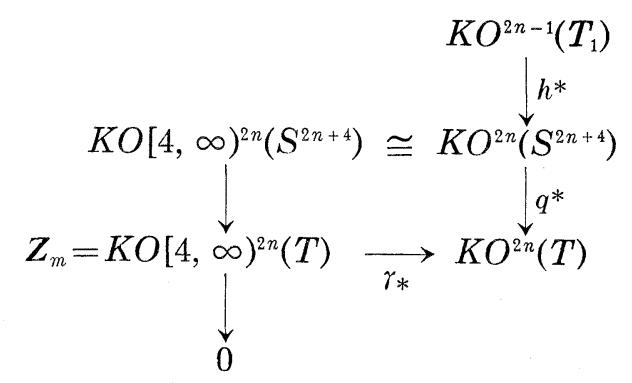


$\gamma_{*}$ is injective and this contradicts (2). Therefore $K O^{2 n-1}\left(T_{1}\right)=Z$, $\operatorname{Im}\left[q^{*}: K O^{2 n-1}\left(S^{2 n+3}\right) \rightarrow K O^{2 n-1}\left(T_{1}\right)\right]=2 K O^{2 n-1}\left(T_{1}\right)$ and $\operatorname{Im}\left[h^{*}: K O^{2 n-1}\left(T_{1}\right) \rightarrow\right.$ $\left.K O^{2 n-1}\left(S^{2 n+3}\right)\right]=(m / 2) K O^{2 n-1}\left(S^{2 n+3}\right)$. This proves (3) and (4).

By 5.3(3), the inclusion induces an injection $K O^{2 n}\left(T_{0}\right) \rightarrow K O^{2 n}\left(S^{2 n} \vee S^{2 n+1}\right)$ $=K O^{2 n}\left(S^{2 n}\right) \oplus K O^{2 n}\left(S^{2 n+1}\right)$ having the image $2 K O^{2 n}\left(S^{2 n}\right) \oplus K O^{2 n}\left(S^{2 n+1}\right)$. Hence $\operatorname{Im}\left[i^{*}: K O^{2 n}\left(T_{0}\right) \rightarrow K O^{2 n}\left(S^{2 n}\right)\right]=2 K O^{2 n}\left(S^{2 n}\right)$. Consider the commutative diagram:

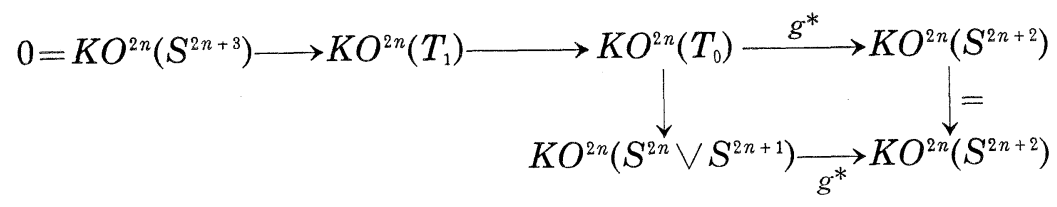

By 5.3(4), the lower $g^{*}$ carries $K O^{2 n}\left(S^{2 n+1}\right)$ isomorphically to $K O^{2 n}\left(S^{2 n+2}\right)$, hence the upper $g^{*}$ carries the torsion subgroup $Z_{2}$ isomorphically to $K O^{2 n}\left(S^{2 n+2}\right)$, therefore $K O^{2 n}\left(T_{1}\right)=Z$ and the inclusion induces an isomorphism $K O^{2 n}\left(T_{1}\right) \cong K O^{2 n}\left(T_{0}\right) / Z_{2}$. By the exact sequence of the pair $\left(T, T_{1}\right)$, it follows that $i^{*}: K O^{2 n}(T) \rightarrow K O^{2 n}\left(T_{1}\right)$ is surjective. This proves (5).

Consider the commutative diagram:

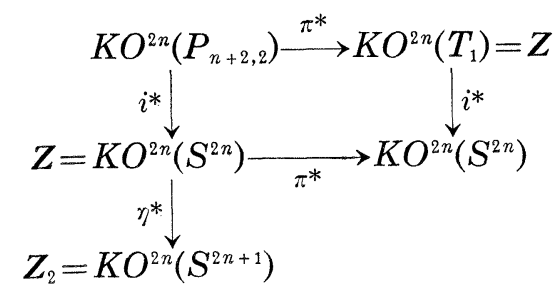

By Fujii [9], $K O^{2 n}\left(P_{n+2,2}\right)=Z$. Since $\eta^{*}$ is surjective, the left $i^{*}$ has the image $2 K O^{2 n}\left(S^{2 n}\right)$. By (5), the right $i^{*}$ has also the image $2 K O^{2 n}\left(S^{2 n}\right)$. By (5.1), the lower $\pi^{*}$ is an isomorphism and hence so is the upper $\pi^{*}$. This proves (6).

By (5.1), (4) and (6), we have the desired diagram of (7).

By (5.1) and (1), we have (8). This completes the proof of 5.8.

We use the notation:

$$
\omega_{i}=r\left(\beta^{i}\left(H_{N}-1\right)\right) \in K O^{-2 i}\left(P_{N}\right),
$$

where $\beta=H_{2}-1 \in K\left(S^{2}\right)$ and $r: K(-) \rightarrow K O(-)$ is the real restriction.

Let $n$ be odd. Then by Fujii [9], we have the exact sequences:

$$
\begin{aligned}
& 0 \longrightarrow K O^{2 n}\left(P_{n+3,3}\right) \longrightarrow K O^{2 n}\left(P_{n+3}\right) \longrightarrow K O^{2 n}\left(P_{n}\right) \longrightarrow 0, \\
& 0 \longrightarrow K O^{2 n}\left(P_{n+2,2}\right) \longrightarrow K O^{2 n}\left(P_{n+2}\right) \longrightarrow K O^{2 n}\left(P_{n}\right) \longrightarrow 0
\end{aligned}
$$

Therefore we identify $K O^{2 n}\left(P_{n+3,3}\right)$ and $K O^{2 n}\left(P_{n+2,2}\right)$ with their images in 
$K O^{2 n}\left(P_{n+3}\right)$ and $K O^{2 n}\left(P_{n+2}\right)$, respectively. The complexification $c: K O^{2 n}\left(P_{n+3}\right)$ $\rightarrow K^{2 n}\left(P_{n+3}\right)$ is injective and hence the Adams operation $\psi^{u}$ on $K O^{2 n}\left(P_{n+3}\right)_{(2)}$ can be computed by the well-known action of $\psi^{u}$ on $K^{2 n}\left(P_{n+3}\right)_{(2)}$. Indeed we can prove the following without difficulties. We omit the details.

LEMMA 5.9. If $n=4 t+k$ with $t \geqq 0$ and $k=1$ or 3 , then

$$
\begin{aligned}
& K O^{2 n}\left(P_{n+3,3}\right) \cong K O^{2 k-8}\left(P_{n+3,3}\right) \\
& =\boldsymbol{Z}\left\{\omega_{4-k} \omega_{0}^{[n / 2]}\right\} \oplus \boldsymbol{Z}\left\{\omega_{4-k} \omega_{0}^{[n / 2]+1}\right\} ; \\
& K O^{2 n}\left(P_{n+2,2}\right) \cong K O^{2 k-8}\left(P_{n+2,2}\right)=Z\left\{\omega_{4-k} \omega_{0}^{[n / 2]}\right\} \text {; } \\
& \operatorname{Im}\left[\alpha^{*}: K O^{2 k-8}\left(S^{2 n+4}\right) \longrightarrow K O^{2 k-8}\left(P_{n+3,3}\right)\right]=Z\left\{\omega_{4-k} \omega_{0}^{[n / 2]+1}\right\} \text {; } \\
& \psi^{u}\left(\omega_{4-k} \omega_{0}^{[n / 2]}\right)=u^{4 t+4} \omega_{4-k} \omega_{0}^{[n / 2]}+(1 / 24)\left(u^{2}-1\right) u^{4 t+4}(n+3) \omega_{4-k} \omega_{0}^{[n / 2]+1} ; \\
& \psi^{u}\left(\omega_{4-k} \omega_{0}^{[n / 2]+1}\right)=u^{4 t+6} \omega_{4-k} \omega_{0}^{[n / 2]+1},
\end{aligned}
$$

where $[n / 2]$ is the greatest integer not exceeding $n / 2$.

Let $z_{1}^{\prime}, z_{2}^{\prime} \in K O^{2 n}\left(P_{n+3,3}\right)$ be the elements corresponding respectively to $\omega_{4-k} \omega_{0}^{[n / 2]}, \omega_{4-k} \omega_{0}^{[n / 2]+1}$ by the Bott isomorphism.

Lemma 5.10. Let $n$ be odd. Then

(1) $K O^{2 n}\left(P_{n+3,3}\right)=\boldsymbol{Z}\left\{\boldsymbol{z}_{1}^{\prime}\right\} \oplus \boldsymbol{Z}\left\{\boldsymbol{z}_{2}^{\prime}\right\}$.

(2) $\operatorname{Im}\left[q^{*}: K O^{2 n}\left(S^{2 n+4}\right) \rightarrow K O^{2 n}\left(P_{n+3,3}\right)\right]=Z\left\{z_{2}^{\prime}\right\}$.

(3) $q^{*}: K O[4, \infty)^{2 n}\left(S^{2 n+4}\right) \cong K O[4, \infty)^{2 n}\left(P_{n+3,3}\right)$, and $\operatorname{Im}\left[K O[4, \infty)^{2 n}\left(P_{n+3,3}\right)\right.$ $\left.\rightarrow K O^{2 n}\left(P_{n+3,3}\right)\right]=\boldsymbol{Z}\left\{z_{2}^{\prime}\right\}$. Hence there exists a unique $z_{2}^{\prime \prime}$ such that $K O[4, \infty)^{2 n}$ $\left(P_{n+3,3}\right)=Z\left\{z_{2}^{\prime \prime}\right\}$ and $z_{2}^{\prime \prime}$ projects to $z_{2}^{\prime}$.

(4) $\quad\left(\psi_{2 n}^{u}-1\right)\left(a z_{1}^{\prime}+b z_{2}^{\prime}\right)=n_{a, b} z_{2}^{\prime}$ and $\psi\left(a z_{1}^{\prime}+b z_{2}^{\prime}\right)=n_{a, b} z_{2}^{\prime \prime}$.

Proof. By 5.9, we have (1), (2).

For simplicity, we set $P=P_{n+3,3}$. Consider the commutative diagram:

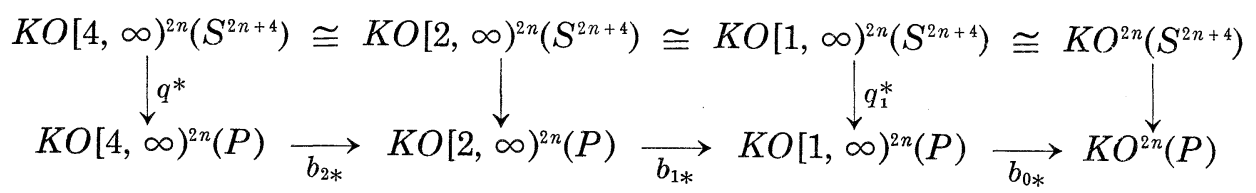

The fibration (4.1) gives a commutative diagram with exact rows:

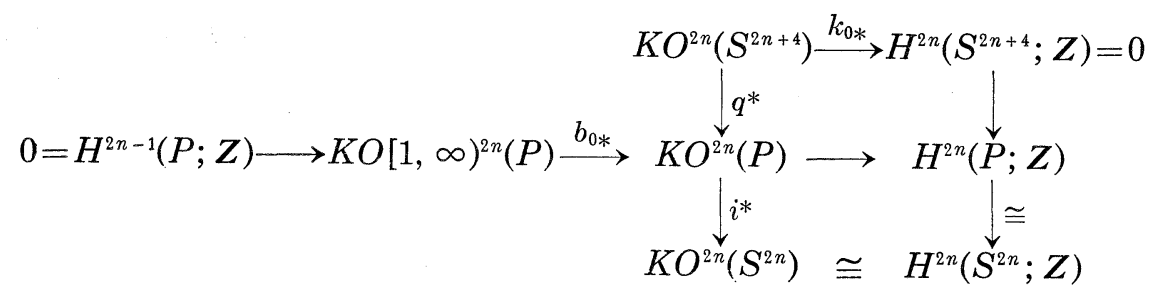


As is easily seen, $\operatorname{Im}\left(i^{*}\right)=2 K O^{2 n}\left(S^{2 n}\right)$. Hence $K O[1, \infty)^{2 n}(P)=Z$ and $\operatorname{Im}\left(b_{0 *}\right)$ $=\boldsymbol{Z}\left\{\boldsymbol{z}_{2}^{\prime}\right\}$ by (1) and (2), and so $q_{1}^{*}$ is an isomorphism. The fibration (4.2) gives an exact sequence

$$
Z_{2}=H^{2 n}\left(P ; Z_{2}\right) \longrightarrow K O[2, \infty)^{2 n}(P) \stackrel{b_{1 *}}{\longrightarrow} K O[1, \infty)^{2 n}(P) \stackrel{k_{1 *}}{\longrightarrow} H^{2 n+1}\left(P ; Z_{2}\right)=0
$$

Thus $K O[2, \infty)^{2 n}(P)$ is $Z$ or $Z \oplus Z_{2}$. The fibration (4.3) gives an exact sequence

$$
0=H^{2 n+1}\left(P ; Z_{2}\right) \longrightarrow K O[4, \infty)^{2 n}(P) \stackrel{b_{2 *}}{\longrightarrow} K O[2, \infty)^{2 n}(P) \stackrel{k_{2 *}}{\longrightarrow} H^{2 n+9}\left(P ; Z_{2}\right) .
$$

As was seen in $\S 4$,

$$
k_{2 *} j_{2 *}=S q^{2}: H^{2 n}\left(P ; Z_{2}\right) \longrightarrow K O[2, \infty)^{2 n}(P) \longrightarrow H^{2 n+2}\left(P ; Z_{2}\right) .
$$

Since $S q^{2}$ is an isomorphism on $H^{2 n}\left(P ; Z_{2}\right)$, it follows that $K O[2, \infty)^{2 n}(P)=$ $Z \oplus Z_{2}$ and $K O[4, \infty)^{2 n}(P)=Z$, so $b_{2 *}$ induces an isomorphism $K O[4, \infty)^{2 n}(P)$ $\cong K O[2, \infty)^{2 n}(P) / Z_{2}$. Therefore $q^{*}: K O[4, \infty)^{2 n}\left(S^{2 n+4}\right) \rightarrow K O[4, \infty)^{2 n}(P)$ is an isomorphism and so

$$
\operatorname{Im}\left[K O[4, \infty)^{2 n}(P) \longrightarrow K O^{2 n}(P)\right]=\operatorname{Im}\left[K O^{2 n}\left(S^{2 n+4}\right) \longrightarrow K O^{2 n}(P)\right]=Z\left\{z_{2}^{\prime}\right\} .
$$

This proves the first part of (3) from which the second part follows easily.

The definition of $\psi_{2 n}^{u}$ gives a commutative square

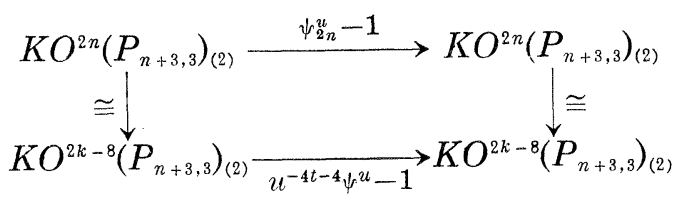

where $n=4 t+k$ with $t \geqq 0$ and $k=1$ or 3 . Hence the first part of (4) follows from 5.9. The second part then follows from (3). This completes the proof of 5.10 .

Now we prove Proposition 5.7. Set

$$
\begin{aligned}
z_{i} & =\pi^{*} z_{i}^{\prime} \in K O^{2 n}(T), \quad i=1,2, \\
z_{2}^{\prime \prime \prime} & =\pi^{*} z_{2}^{\prime \prime} \in K O[4, \infty)^{2 n}(T) .
\end{aligned}
$$

Then (1) follows from 5.8(5). We have (2) by 5.8(1), (8) and 5.10(3). We have (3) by 5.8(7), 5.9 and 5.10(1), (2). Applying $\pi^{*}$ to the equations in 5.10(4), we obtain (4). This completes the proof of 5.7 . 


\title{
References
}

[1] J. F. Adams, On the groups J (X)-II, Topology, 3 (1965), 137-171.

[2] J. F. Adams, On the groups J (X)-III, Topology, 3 (1965), 193-222.

[ 3 ] J. C. Becker and D. H. Gottlieb, Transfer maps for fibrations and duality, Comp. Math., 33 (1976), 107-133.

[4] M. C. Crabb, Z/2-homotopy theory, London Math. Soc. Lecture Notes 44, Camb. Univ. Press, Cambridge, 1980.

[5] M. C. Crabb and K. Knapp, Vector bundles of maximal codegree, Math. Z., 193 (1986), 285-296.

[6] M. C. Crabb and K. Knapp, James numbers and the codegree of vector bundle, preprint.

[ 7 ] M. C. Crabb and K. Knapp, James numbers, Math. Ann., 282 (1988), 395-422.

[ 8 ] I. Dibag, Degree theory for spherical fibrations, Tôhoku Math. J., 34 (1982), 161177.

[9] M. Fujii, $K_{0}$-groups of projective spaces, Osaka J. Math., 4 (1967), 141-149.

[10] P. J. Kahn, Mixing homotopy types of manifolds, Topology, 14 (1975), 203-216.

[11] P. May, $E_{\infty}$ ring spaces and $E_{\infty}$ ring spectra, Lecture Notes in Math., 577, Springer-Verlag, Berlin, 1977.

[12] H. Ōshima, On the stable James numbers of complex projective spaces, Osaka J. Math., 11 (1974), 361-366.

[13] H. Ōshima, Some James numbers of Stiefel manifolds, Math. Proc. Cambridge Philos. Soc., 92 (1982), 139-161.

[14] M. Rothenberg, The $J$ functor and the nonstable homotopy groups of the unitary groups, Proc. Amer. Math. Soc., 15 (1964), 264-271.

[15] R. Tanaka, On the stable James numbers of Thom complexes, Osaka J. Math., 20 (1983), 137-143.

[16] E. Thomas, Homotopy classification of maps by cohomology homomorphisms, Trans. Amer. Math. Soc., 111 (1964), 138-151.

[17] G. Walker, Estimates for the complex and quaternionic James numbers, Quart. J. Math., 32 (1981), 467-489.

[18] I. Yokota, Groups and topology, Shohkaboh, Tokyo, 1971.

\author{
Department of Mathematics \\ OSAKA CITY UNIVERSITY \\ Sugimoto, SUMIYoShI-KU \\ OSAKA 558, JAPAN
}

